\title{
COMMUNICATIONS
}

\section{PERIPHERAL RETINAL ANGIOMA WITH EXUDATIVE RETINOPATHY IN ADULTS (COATS'S LESION)*}

\author{
BY \\ PAUL HENKIND $\dagger$ AND GWYN MORGAN \\ From the Department of Pathology, Institute of Ophthalmology, University of London
}

IN 1908, Coats, in his classic paper entitled "Forms of Retinal Disease with Massive Exudation", discussed three entities: (1) Cases without gross vascular disease of the retinal vessels. (2) Cases with gross vascular disease. (3) "A very peculiar group ... which is characterized by the formation of large arteriovenous communications." These cases are now considered to be identical with von Hippel's disease or retinal angiomatosis, and the two former groups are termed Coats's disease, types 1 and 2 respectively. Unfortunately, there exists a good deal of confusion about these two entities. Clinically, Coats's disease is rarely seen from its inception to its ultimate conclusion; it has been difficult to obtain early lesions for histological examination; and, finally, the pathogenesis of the disorder is still speculative.

Most authorities agree about certain clinical features which may be of use in the diagnosis of this disease of unknown aetiology:

Generally affects younger persons ( 20 per cent. older than 21 years).

Males are more commonly involved than females.

Usually unilateral (bilateral $10-15$ per cent.).

Usually no related systemic disorder.

No early anterior segment ocular disease.

Non-familial.

The ocular findings, particularly in early cases, are fairly specific:

Type 1

Deep exudative mass generally at posterior pole.

Usually a "woolly" or "flocculent" appearance.

Yellow to greyish-green exudate.

Exudate raised 1.5-4.5 D, always beneath retinal vessels.

Glistening spots and areas suggestive of cholesterol.

Concomitant retinal detachment.

Retinal haemorrhages.

Retinal vessels usually appear normal.

\footnotetext{
* Received for publication March $19,1965$.
}

† Special Fellow No. 1F 11 NB 1128-01 of the Division of Neurological Disease and Blindness, National Institute of Health, United States Public Health Service. On leave from the Department of Ophthalmology, New York University College of Medicine. 
Type 2

As Type 1, plus following vascular changes:

Irregular vessel calibre.

Vascular sheathing.

Vessel anastomoses.

Aneurysms.

Neovascularization.

In the later stages the picture may be obscured by a variety of secondary changes. Not infrequently, the Department of Pathology at the Institute of Ophthalmology, London, receives eyes which are suspected of harbouring a retinoblastoma, and which on histological examination prove to be cases of Coats's disease. Apart from the clinical aspects, there are certain histological criteria which allow one to make a presumptive diagnosis of the disorder:

Abnormal retinal vessels-dilated capillaries.

Exudate in deep retinal layers.

Retinal haemorrhage and gliosis.

Intra-retinal cyst formation and retinal detachment.

Organization of haemorrhage with lipoidal macrophages

("ghost" cells), and cholesterol clefts in sub-retinal space.

In Type 1 no gross vascular changes are noted, but in Type 2 perivasculitis, telangiectases, and aneurysms are common.

This communication is concerned with four older patients, all of whom had undergone enucleation because of severe ocular disease, and whose eyes, on histological examination, showed the features associated with Coats's disease, i.e., deep retinal exudation, lipoidal macrophages or "ghost cells", and cholesterol clefts in the subretinal exudate. In none of the cases was the diagnosis of Coats's disease entertained clinically. Apart from the usual histological features, each eye harboured a small but pronounced vascular malformation at the retinal periphery, and these will be discussed in detail.

\section{Case Reports}

Details of the clinical features in the four cases are given in the Table.

TABLE

Clinical features of the four Cases

\begin{tabular}{c|c|l|l}
\hline Age & Sex & \multicolumn{1}{|c}{ Presenting Sign } & \multicolumn{1}{c}{ Reason for Enucleation } \\
\hline 71 & F & Left eye-chronic narrow-angle glaucoma & Malignant glaucoma \\
68 & M & Left eye-retinal detachment at age of 52 & $\begin{array}{l}\text { Blind left eye } \\
\text { Glaucoma and keratic precipitates } \\
\text { right eye }\end{array}$ \\
54 & M & Left eye-solid retinal detachment & ? Malignant melanoma \\
64 & F & Left eye-exudative detachment at age of 61 & Glaucoma \\
\hline
\end{tabular}


Case 1.-A 71-year-old female was diagnosed in 1961 as having chronic narrow-angle glaucoma in her left eye, and since then her vision had deteriorated. In 1964 a Scheie procedure was performed and this was followed by malignant glaucoma which was unresponsive to treatment. The eye was removed.

The patient is alive and well, without any evidence of vascular malformations, and there is no family history of similar eye disease.

\section{Pathological Examination.}

Macroscopical appearance.-There was a superior iridectomy and a shallow anterior chamber. The lens was partially opaque and there appeared to be some exudate covering the inferior portion of the retina.

Microscopical appearance (Figs 1-3).-A small elevated vascular malformation of the retina was present at the ora serrata, and in this region the retina showed several thick and numerous thin-walled vessels. Occasional lipoidal cells were seen beneath the endothelium, and both reticulin and collagen fibres were demonstrated in the larger vessel walls. Retinal gliosis, chronic inflammatory-cell infiltration, and pigment epithelium proliferation were present in the neighbourhood of the malformation, and the subretinal space in this area contained organized haemorrhagic

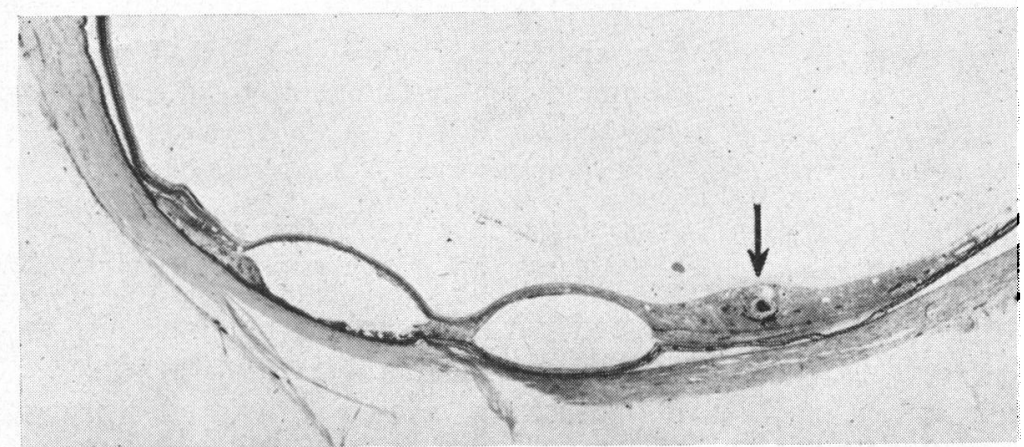

Fig. 1.-Case 1. Low power photomicrograph showing the thickened, partially detached retina, with a vascular malformation at the periphery (arrow). H. and E.

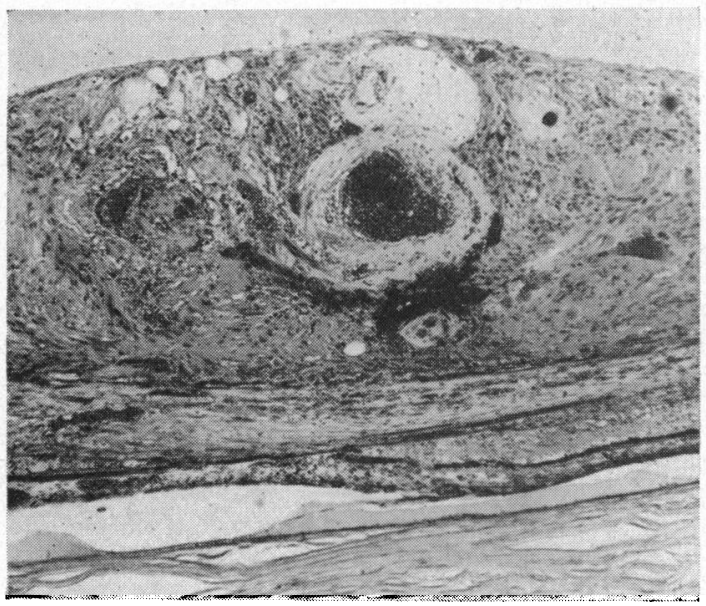

FIG. 2. Case 1. Higher power view of the vascular malformation in Fig. 1, showing dilated thick and thin-walled vessels, with pigment proliferation and gliosis. Lipoidal macrophages and organized exudate can be seen in the subretinal space. H. and E. $\times 50$.

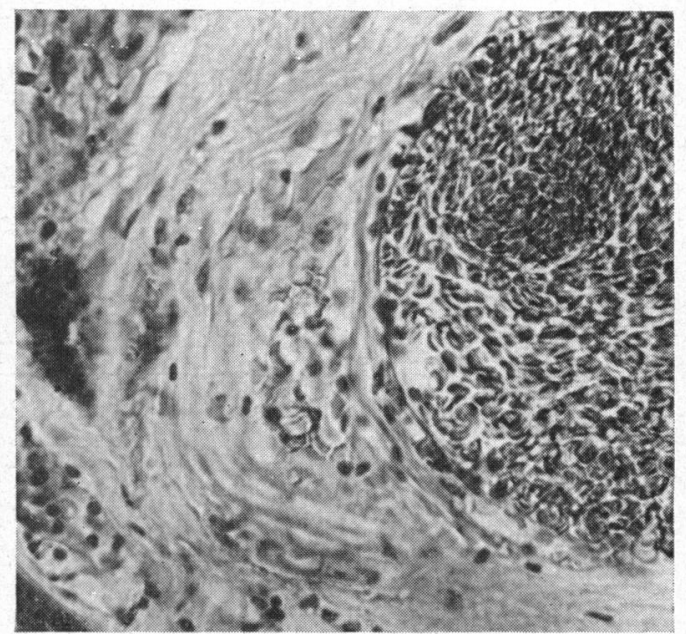

Fig. 3.-Case 1. Higher power view of the wall of one of the vessels in Fig. 2, showing marked thickening and lipoidal macrophages. H. and E. $\times 300$. 
exudate with pigmented lipoidal macrophages, cholesterol clefts, occasional giant cells, and prominent fibrosis. The retina posterior to the lesion was detached and the subretinal exudate contained pigmented macrophages.

Other features were a surgical iridectomy, peripheral anterior synechiae, a fibrovascular membrane on the anterior iris surface, the iris adhering to the cataractous lens, peripheral cystic retinal degeneration, and gliosis of the optic nerve.

Case 2.-A 68-year-old male had noted, at the age of 52, diminished vision in his left eye. A retinal detachment was diagnosed and treated by immobilization in bed for five months. The vision gradually diminished and was totally lost at the age of 68 . In addition to the detachment, the left eye developed a hypermature cataract with extrusion of lens material into the anterior chamber, and glaucoma was noted in the right eye. When keratic precipitates became evident in the right eye, enucleation of the blind left eye was performed.

There was no evidence of angiomata in the right eye or elsewhere, and there was no family history of similar ocular disease or angiomatosis.

Pathological Examination.

Macroscopical appearance.-The retina was detached and the albuminous subretinal exudate contained cholesterol crystals.

Microscopical appearance (Figs 4-7).-A small elevated vascular malformation of the retina was present at the ora serrata; in this area a number of enlarged and dilated, thick, and occasionally thin-walled, vessels filled with pinkish exudate occupied almost the entire thickness of the retina.

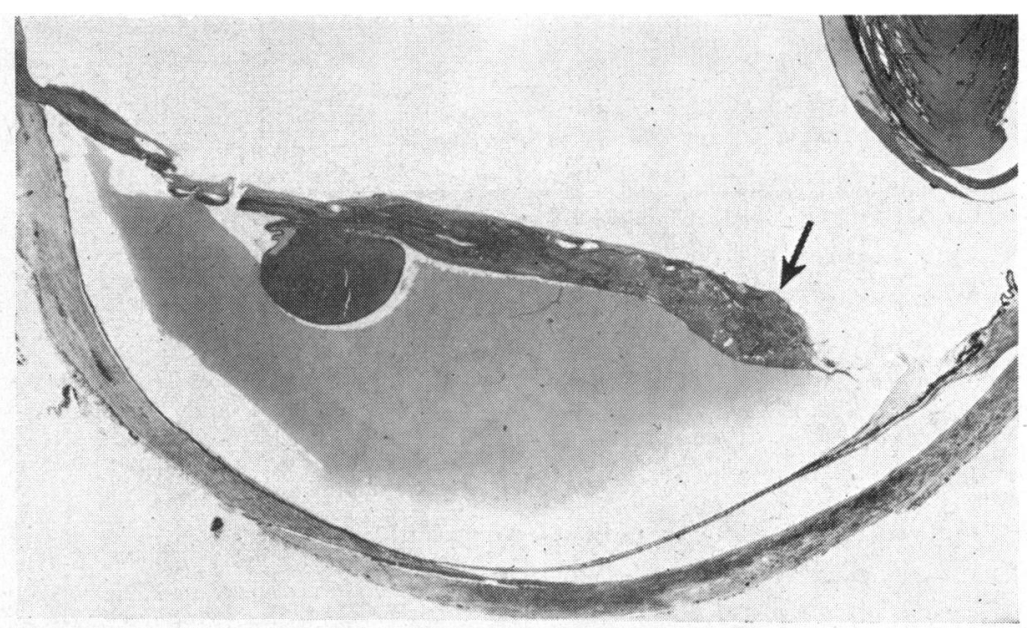

FIG. 4.-Case 2. Low power photomicrograph showing the thickened retina with the vascular malformation at the periphery (arrow), a large cyst posteriorly, and subretinal exudate. $H$. and $E$.

The larger vessels had lipoidal cells in their walls, as well as similar cells lining the lumen. A marked degree of pigment epithelium proliferation was present around the vessels, as well as some gliosis, and a small amount of eosinophilic exudate was present beneath the malformation. The vessel walls contained much reticulin and collagen, but there was little reticulin between the vessels. The subretinal space contained lipoidal macrophages within the albuminous exudate, and on the opposite side pigmented macrophages were present. Posteriorly, the retina was thickened and a large blood-filled cyst of the deeper retinal layers was apparent. On the opposite side the retina was detached, and subretinal fibrosis was prominent.

Other features were rubeosis iridis, uveitis, peripheral retinitis proliferans, cataract, ringschwiele, and gliosis of the optic nerve. 


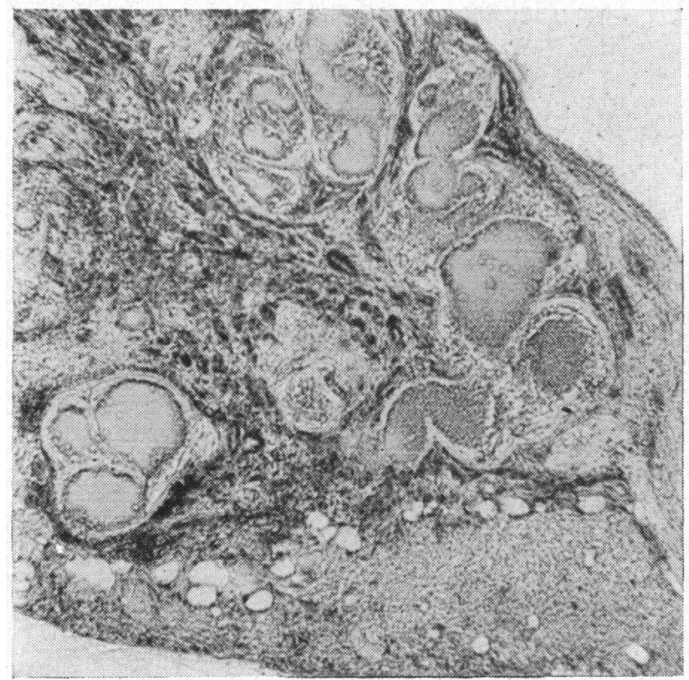

FIG. 5.-Case 2. Higher power view of the vascular malformation in Fig. 4, showing numerous dilated vascular channels, marked pigment proliferation, and areas of gliosis. H. and E. $\times 45$.

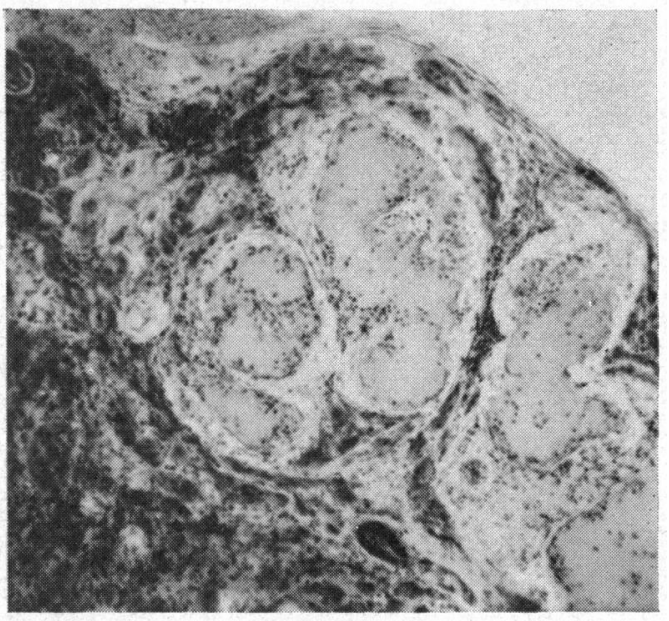

FIG. 6.-Case 2. A higher power view of Fig. 5 showing dilated thick-walled vascular channels and pigment proliferation. H. and E. $\times 75$.

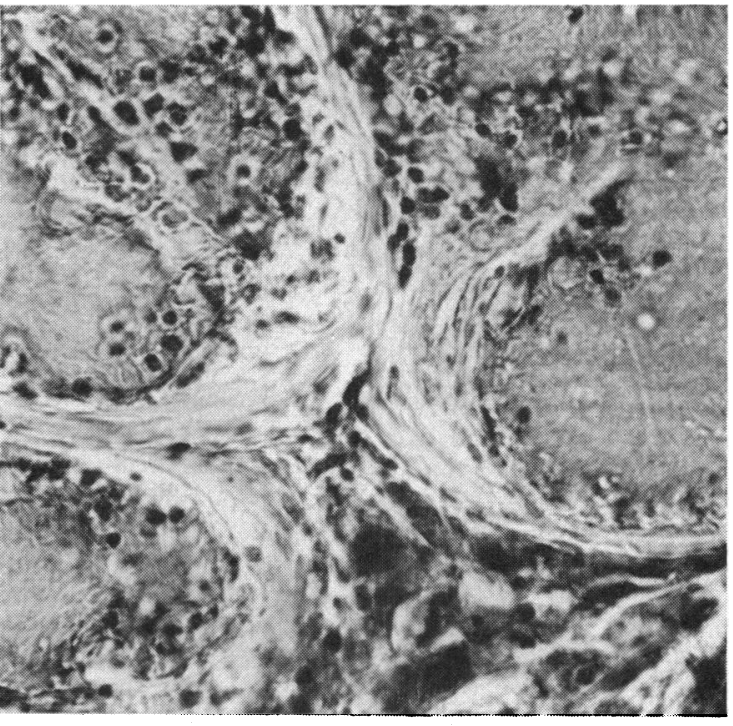

FIG. 7.-Case 2. A higher power view of Fig. 6, showing thick-walled vessels lined by lipoidal macrophages. H. and E. $\times 335$. 
Case 3.-A 54-year-old male noted defective vision in his left eye five weeks prior to enucleation. A large solid retinal detachment occupying the temporal quadrants was present and a malignant melanoma of the choroid was considered the likely diagnosis.

Pathological Examination.

Macroscopical appearance.-The retina was detached and there was subretinal albuminous exudate containing cholesterol crystals. No intra-ocular new growth was evident.

Microscopical appearance (Figs 8 and 9). - There was a small angiomatous malformation of the retina near the ora serrata, composed of numerous dilated and thick-walled vascular channels. Haemorrhage was present in the deeper retinal layers beneath the tumour, and there was haemorrhagic subretinal exudate laden with pigment containing "ghost cells" and cholesterol clefts. The remaining retina on this side was thickened and disorganized, containing multiple pockets of

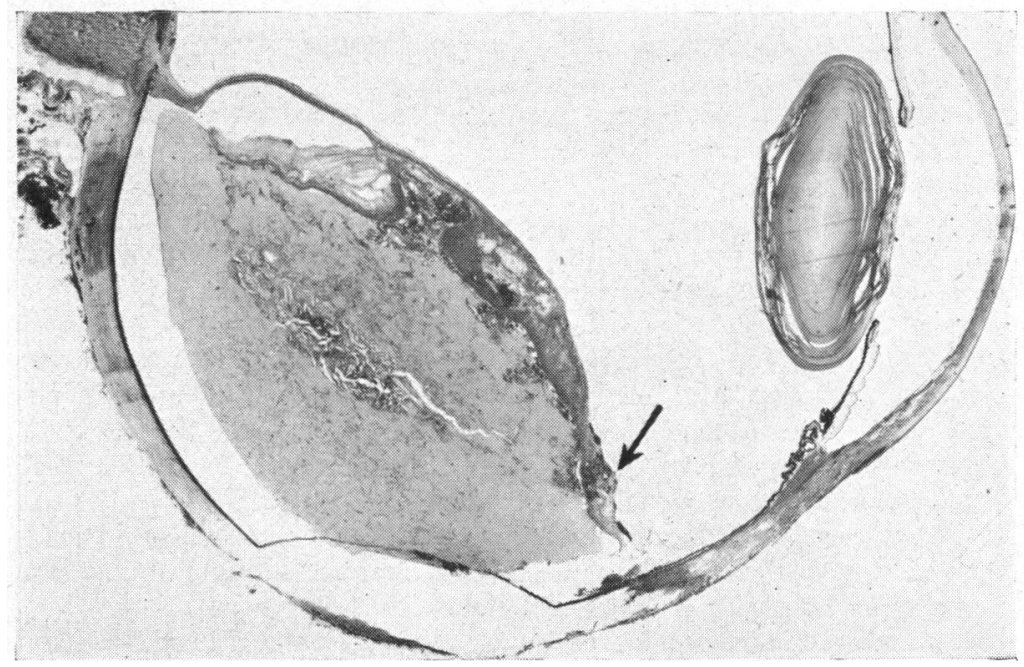

Fig. 8.-Case 3. Low power photomicrograph showing the thickened cystic retina, which contains a peripheral vascular malformation (arrow) and subretinal exudate containing numerous cholesterol clefts. $H$. and $\mathrm{E}$.

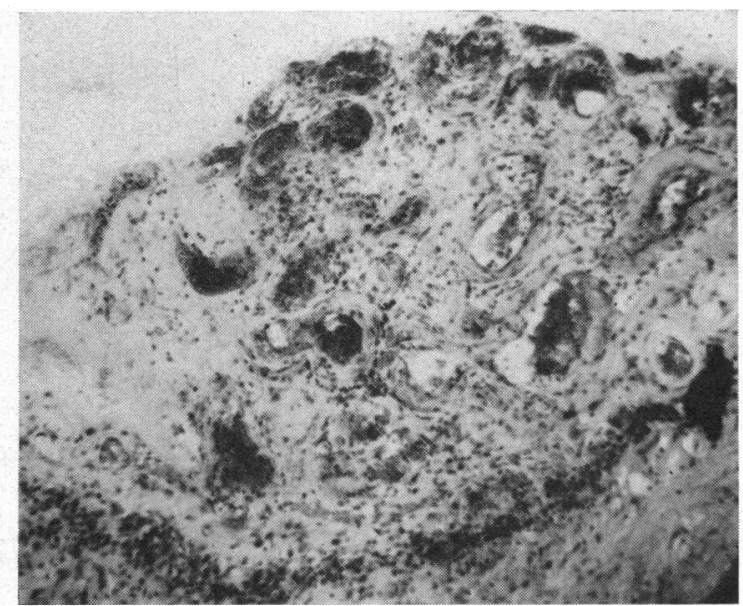

FIG. 9.-Case 3. Higher power view of the vascular malformation in Fig. 8, showing numerous dilated thick and thin-walled vascular channels. H. and E. $\times 50$. 
orange-pink exudate, dilated vascular channels, and a large cyst filled with pink fibrillar coagulum. Choroidoretinal adhesions and proliferation of the pigment epithelium were also present. On the side opposite the vascular malformation there was a peripheral retinal detachment with pigment cell and cholesterol cleft containing subretinal exudate, as well as a shallow posterior pole detachment and choroidoretinal adhesions and atrophy. There was a small amount of haemorrhage in the vitreous cavity.

Other pathological features were a healed, non-perforating, corneal wound, mild rubeosis iridis, ectropion uveae, and mild anterior uveitis.

Case 4.-A 64-year-old female had a left intracapsular cataract extraction at the age of 58. Three years later an exudative retinal detachment was noted in this eye, with the suggestion of a horseshoe tear in the involved area; surgery was declined. After a lapse of three years the patient presented with a bullous retinal detachment infero-temporally; large feeding vessels could be seen in the affected region, and a diagnosis of haemangioblastoma was made. The ocular tension increased and the eye was enucleated.

The patient has been lost to follow-up, but there was no evidence of an angiomatous malformation in her right eye or elsewhere in the body. Following enucleation the patient suffered occasional bouts of giddiness or "blackouts".

Pathological Examination.

Macroscopical appearance.-The globe was slightly collapsed, the lens was absent, and the vitreous clear. The retina was detached inferiorly, and clear subretinal albuminous exudate was present.

Microscopical appearance (Fig. 10).--Situated at the retinal periphery within its inner layers there was a circumscribed nodule of angiomatous tissue, consisting of multiple, closely packed, sinusoidal spaces lined by endothelial cells and filled with blood. Some lipoidal cells were present in the vessel walls which contained reticulin and collagen. The retina was almost completely detached, and it was thickened inferiorly by diffuse gliosis; anteriorly the thickness increased markedly, and many small cystic spaces were present within the vascularized glial tissue. Pinkish exudate was evident throughout the involved retina, and the subretinal exudate contained lipoidal macrophages and occasional cholesterol clefts.

Other features were anterior synechiae occluding the angle, and a partially atrophic iris with rubeosis and ectropion uveae.

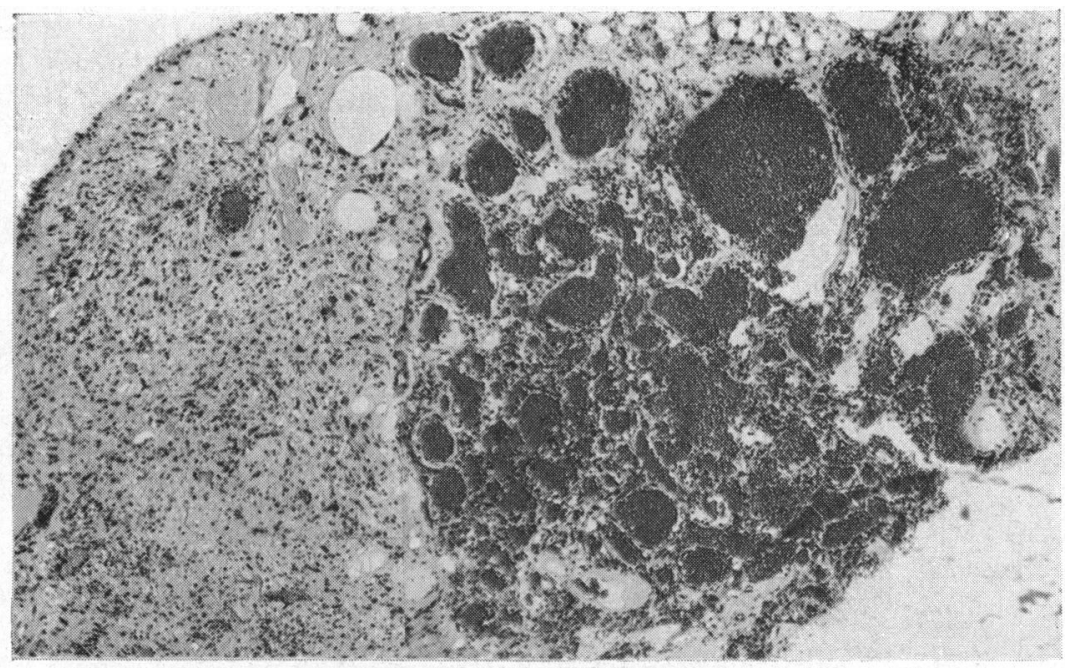

Fig. 10.-Case 4. Photomicrograph showing a cavernous angiomatous malformation composed of numerous thin-walled channels, and the adjacent retina, which shows reactive gliosis and cystic degeneration. H. and $\mathrm{E}, \quad \times 45$, 


\section{Discussion}

The four patients presented in this paper, who were all over 50 years of age at the time of their initial ocular symptoms, were in good general health, and had no family history of similar ocular disease. All four eyes showed severe, diffuse, uni-ocular retinal disease, associated with localized malformations of the retinal vessels at the periphery.

Pathologically, all four eyes appear to satisfy the histological criteria of Coats's disease type 2-namely, exudative retinopathy with gross localized disease of the retinal vessels. The lesion in the fourth case may be diagnosed histologically as a cavernous angioma, although clinically the patient presented with features which are generally associated with von Hippel's disease, i.e., an elevated retinal mass with feeding vessels. Similar vascular lesions have been considered to be an integral part of Coats's disease when associated with deep retinal exudation, lipoidal macrophages, and cholesterol clefts in the subretinal exudate (Hogan and Zimmerman, 1962, Fig. 462).

The fact that vascular malformations at the retinal periphery were detected at all in the first three cases was completely fortuitous, for they were neither clinically evident nor apparent on gross examination of the opened eye. Moreover, serial sections revealed the rather small extent of these lesions, and in the fourth case multiple sections had to be cut before the clinically suspected malformation was found. It would not be surprising, therefore, if similar cases have been overlooked both by ophthalmologists and ocular pathologists.

It is unlikely that these cases were of von Hippel's disease, for although the vascular anomalies occurred at the most common site for angiomatosis retinae, namely, the retinal periphery, and in the last case feeding vessels were seen leading to the detached retina, the late onset of the disease is not in favour of the diagnosis. Joe and Spencer (1964), however, described the disease in a 53-year-old male.

The usually accepted histological criteria for von Hippel's disease were not seen in any of our four cases; angioblastic endothelial cells, pseudoxanthoma cells, and an intervascular reticulin network were all absent. It can be argued, of course, that the type of angioma seen in von Hippel's disease is only a variant of a wide range of vascular anomalies. For purposes of classification, however, we feel that the histological features of our four cases would not be generally accepted for the diagnosis of von Hippel's disease. It seems likely that they represent old and quiescent vascular anomalies, which have lost their early characteristics and are now associated with gliosis.

It is not possible to be certain of the pathogenesis of these lesions. In Case 1, the thick- and thin-walled dilated vascular channels may possibly have resulted from peripheral stasis, for somewhat similar histological features were noted by Ashton (1965) in a case of thrombotic glaucoma (Fig. 11); a pre-existing vascular anomaly in Case 1, however, cannot be ruled out. Both the second and third cases showed multiple, greatly dilated, vascular channels lying in a matrix of distorted and gliosed retinal tissue. Whether or not these channels were initially telangiectatic vessels, which Reese (1963) has suggested as the underlying pathogenesis of 


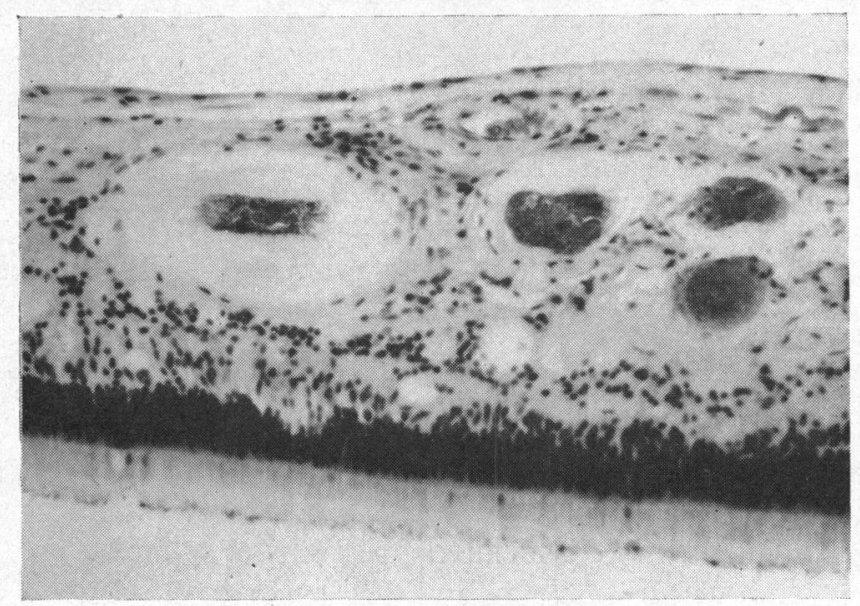

FIG. 11.-Photomicrograph of a section of retina from a case of thrombotic glaucoma, showing four dilated thick-walled vascular channels somewhat similar to those seen in Case 1 . Note that the retina is only slightly thickened. H. and E. $\times 110$.

Coats's disease, or secondarily affected normal vascular channels, is not known, but the former explanation seems more likely. The last case is somewhat puzzling; no retinal vascular changes were noted at the time when an exudative retinal detachment was initially diagnosed, yet three years later large feeding vessels led to the detached area and suggested the clinical diagnosis of retinal haemangioblastoma.

As already mentioned we do not feel that the histological features of this case were compatible with the diagnosis of von Hippel's disease. We consider it likely that the vascular anomalies in all four cases existed from birth and were activated much later, perhaps because of local stasis and hypoxia. Leakage of blood and protein from these abnormal vessels may well have resulted in the histological appearances of an exudative retinopathy.

Reese (1963) limits the diagnosis of Coats's disease to young patients with the classic clinical findings already described. We do not subscribe to this view, hbwever, for cases of Coats's disease have been described in adults (Woods and Duke, 1963), in which the clinical and histopathological features were identical with those seen in Coats's disease affecting young patients. It appears, however, that this condition is less common after the third decade of life, and it is possible that the precipitating or initiating factors may differ in the adult and juvenile cases.

We should like to suggest that this form of exudative retinopathy should be labelled "Coats's lesion" rather than his disease, because it appears to be more of a reaction than a specific entity. Moreover, we feel that the pathogenesis of the lesion is the same irrespective of the patient's age, the exudative features being associated with a variety of abnormal, dilated, leaking, vascular channels. 


\section{Summary}

The eyes from four elderly patients have been described, all being removed because of severe ocular disease. None was diagnosed clinically as Coats's disease, but the histological appearances were typical of this entity. We believe that the pathological picture probably derived from the well-defined vascular anomalies found at the periphery of the retina in each case, resulting in Coats's lesion, and we consider that the pathogenesis in no way varies from that of juvenile Coats's disease.

We should like to thank Prof. Norman Ashton for his invaluable advice, and we are grateful to Miss E. FitzGerald for secretarial help, and to Mr. V. J. Elwood and Mr. J. Peacock for their technical assistance.

\section{REFERENCES}

Ashton, N. (1965). Personal communication.

COATs, G. (1908). Roy. Lond. ophthal. Hosp. Rep., 17, 440.

Hogan, M. J., and Zimmerman, L. E. (1962). "Ophthalmic Pathology", 2nd ed., p. 533. Saunders, Philadelphia.

Joe, S., and SPENCER, W. H. (1964). Arch. Ophthal. (Chicago), 71, 508.

REESE, A. (1963). "Tumors of the Eye", 2nd ed., p. 377. Hoeber, New York.

Woods, A. C., and Duke, J. R. (1963). Brit. J. Ophthal., 47, 385. 